\title{
Nonomuraea kuesteri sp. nov.
}

Correspondence
Peter Kämpfer
peter.kaempfer@agrar.
uni-giessen.de

\author{
Peter Kämpfer, ${ }^{1}$ Reiner M. Kroppenstedt ${ }^{2}$ and Iris Grün-Wollny ${ }^{3}$ \\ ${ }^{1}$ Institut für Angewandte Mikrobiologie, Justus-Liebig-Universität Giessen, D-35392 Giessen, \\ Germany \\ ${ }^{2}$ DSMZ - Deutsche Sammlung von Mikroorganismen und Zellkulturen GmbH, D-38124 \\ Braunschweig, Germany \\ ${ }^{3}$ Labor Grün-Wollny, D-35394 Giessen, Germany
}

The genus Nonomuria [sic, corrected by Chiba et al. (1999) to Nonomuraea] was originally proposed by Zhang et al. (1998) as a member of the family Streptosporangiaceae that forms extensively branched substrate and aerial mycelia. On the basis of detailed phylogenetic analysis, the genus presently comprises 18 species and two subspecies, most of which were listed by Gyobu \& Miyadoh (2001), Stackebrandt et al. (2001) and Quintana et al. (2003).

During the characterization of organisms from soil, strain GW $14-1925^{\mathrm{T}}$ was recovered on oatmeal agar (ISP 3 medium; Küster, 1959) at $25^{\circ} \mathrm{C}$; it had slightly yellowcoloured vegetative mycelium with white aerial mycelium. Subcultivation was done on tryptone soy agar (Oxoid) at $25{ }^{\circ} \mathrm{C}$ for $24 \mathrm{~h}$. Gram-staining was performed as described by Gerhardt et al. (1994). The cell morphology was observed under a Zeiss light microscope at $\times 1000$, using cells that had been grown for 3 days at $25^{\circ} \mathrm{C}$ on R2A agar (Oxoid). The 16S rRNA gene was analysed as described previously (Kämpfer et al., 2003). Phylogenetic analysis was performed

Published online ahead of print on 29 October 2004 as DOI 10.1099/ ijs.0.63380-0.

The GenBank/EMBL/DDBJ accession number for the 16S rRNA gene sequence of strain DSM $44753^{\top}$ is AJ746362. by using the ARB software package (Strunk et al., 2000) and also the software package MEGA, version 2.1 (Kumar et al., 2001), after multiple alignment of the data by CLUSTAL_X (Thompson et al., 1997).

Distances were obtained (using distance options according to the Kimura two-parameter model; Kumar et al., 2001) and clustering was performed, using the neighbour-joining (Fig. 1) and maximum-parsimony methods, by using bootstrap values based on 1000 replications. The 16S rRNA gene sequence of strain GW $14-1925^{\mathrm{T}}$ was a continuous stretch of $1496 \mathrm{bp}$. Sequence similarity calculations after neighbour-joining analysis indicated that the closest relatives of strain GW $14-1925^{\mathrm{T}}$ were Nonomuraea turkmeniaca $(98.9 \%)$, Nonomuraea helvata (98.6\%), Nonomuraea salmonea (98.3\%) and Nonomuraea polychroma (98.5\%). Both the neighbour-joining tree (Fig. 1) and the maximumparsimony tree (not shown) revealed that strain GW 14$1925^{\mathrm{T}}$ clustered most closely with Nonomuraea roseoviolacea subsp. roseoviolacea $(98 \cdot 1 \%)$ and Nonomuraea longicatena $(97 \cdot 9 \%)$.

The results of chemotaxonomic analyses are given in the species description below. The following characteristics were analysed: menaquinones (as described by Kroppenstedt, 1985), polar lipids (as described by Lechevalier et al., 1977; 


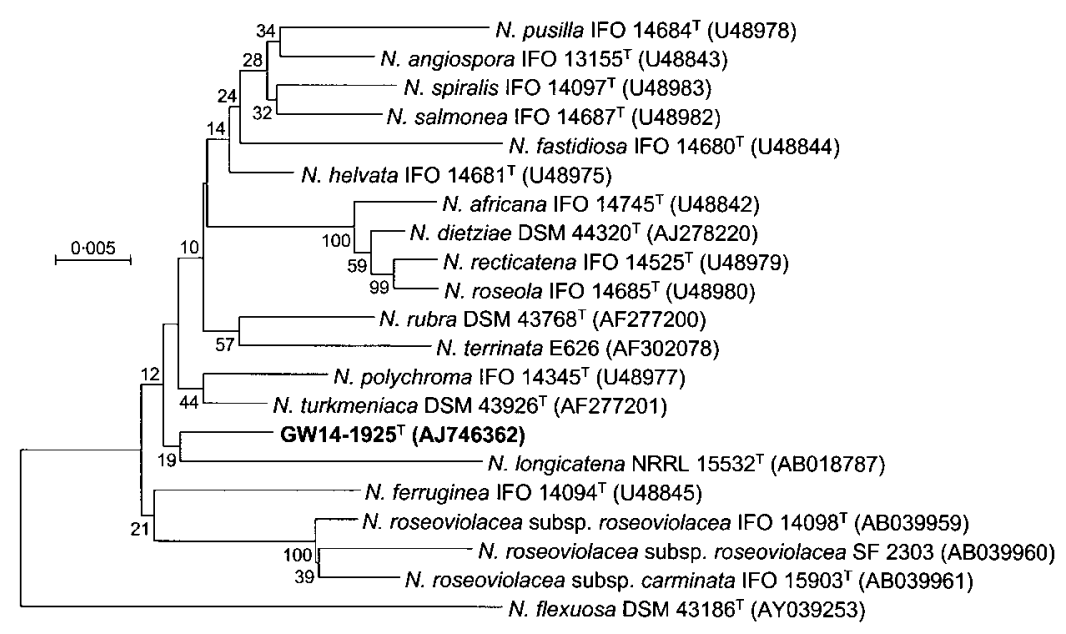

Fig. 1. Phylogenetic analysis, based on $16 \mathrm{~S}$ rRNA gene sequences available from the EMBL database (accession nos are given in parentheses), constructed after multiple alignment of data by CLUSTAL_X (Thompson et al., 1997). Distances were obtained (using distance options according to the Kimura-2 model) and clustering was performed, using the neighbour-joining method, by using the software package MEGA, version 2.1 (Kumar et al., 2001). Bootstrap values based on 1000 replications are listed as percentages at branching points. Bar, $0.005 K_{\text {nuc }}$.
Minnikin et al., 1984) and fatty acids (as described by Kämpfer \& Kroppenstedt, 1996).

The quinone system found supports affiliation of strain GW $14-1925^{\mathrm{T}}$ to the genus Nonomuraea. The principal menaquinones of strain GW $14-1925^{\mathrm{T}}$ were as follows: MK-9 $\left(\mathrm{H}_{4}\right), 71 \%$; MK-9 $\left(\mathrm{H}_{6}\right), 15 \%$; MK-9(H $\left.\mathrm{H}_{2}\right), 6 \%$; and MK-9, $2 \%$. In addition, MK-8 $\left(\mathrm{H}_{4}\right)$ was detected $(6 \%)$. This is essentially in accordance with the quinone profile reported for members of the genus (Kroppenstedt \& Goodfellow, 1991; Stackebrandt et al., 2001; Quintana et al., 2003). The polar lipids of strain GW $14-1925^{\mathrm{T}}$ are of the PIV type, according to the phospholipid classification of Lechevalier et al. (1977), and include diphosphatidylglycerol, phosphatidylinositol, phosphatidylethanolamine, methylphosphatidylethanolamine and the diagnostic phospholipid $\mathrm{N}$-acetylglucosamine. Some unidentified glycolipids were also detected. The fatty acids comprised mainly iso- and 10-heptadecanoic-branched fatty acids. Smaller amounts of unbranched saturated and 2-hydroxy fatty acids were detected (fatty acid type 3c of Kroppenstedt, 1985). The detailed fatty acid profile is as follows: $13: 0(0 \cdot 4 \%), 14: 0$ iso $(0 \cdot 9 \%), 14: 0(0 \cdot 9 \%), 15: 1$ iso $\mathrm{G}(0 \cdot 2 \%), 15: 0$ iso $(7 \cdot 9 \%), 15: 0$ anteiso $(0 \cdot 3 \%), 15: 0(3 \cdot 8 \%), 16: 1$ iso $\mathrm{G}$ $(8 \cdot 3 \%), 16: 0$ iso $(28 \cdot 9 \%), 16: 1$ cis $9(1 \cdot 8 \%), 16: 0(1 \cdot 8 \%)$, $15: 0$ 2-OH $(0 \cdot 8 \%), 16: 0$ 10-methyl $(2 \cdot 8 \%), 17: 0$ iso $(1 \cdot 8 \%), 17: 0$ anteiso $(1 \cdot 0 \%), 17: 1$ cis $9(4 \cdot 2 \%), 16: 0$ iso 2-OH (10.5\%), $17: 0(1 \cdot 6 \%), 16: 02-\mathrm{OH}(0 \cdot 5 \%), 17: 010-$ methyl $(15 \cdot 5 \%), 18: 0$ iso $(0 \cdot 6 \%), 18: 1$ cis $9(0 \cdot 6 \%), 17: 0$ iso $2-\mathrm{OH}(0 \cdot 4 \%), 18: 0(1 \cdot 5 \%), 17: 02-\mathrm{OH}(0 \cdot 3 \%)$ and 18:0 10-methyl $(0 \cdot 9 \%)$. The results of the physiological characterization, performed using methods described previously (Kämpfer, 1990; Kämpfer et al., 1991), are given in Table 1 and in the species description. In addition, degradative tests were performed according to Williams et al. (1983). DNA-DNA hybridizations between strain GW $14-1925^{\mathrm{T}}$ and the type strains of $N$. longicatena, $N$. salmonea and $N$. turkmeniaca were performed using the method described by Ziemke et al. (1998), except that, for nick translation, $2 \mu \mathrm{g}$ DNA was labelled during a $3 \mathrm{~h}$ incubation at $15^{\circ} \mathrm{C}$. Strain GW $14-1925^{\mathrm{T}}$ showed relatively low
DNA-DNA relatedness to $N$. roseoviolacea subsp. roseoviolacea DSM $43144^{\mathrm{T}}(15 \cdot 9 \%$, mean value of four hybridizations), N. longicatena NRRL $15532^{\mathrm{T}}$ (16\%, mean value of two hybridizations), N. salmonea DSM $43678^{\mathrm{T}}(32 \%$, mean value of four hybridizations) and $N$. turkmeniaca DSM $43926^{\mathrm{T}}(40 \cdot 5 \%$, mean value of four hybridizations). It has been shown that Nonomuraea species have high $16 \mathrm{~S}$ rRNA gene sequence similarities (within the range 97.6-99.4\%) and have low DNA-DNA relatedness values (Fischer et al., 1983; Poschner et al., 1985; Tamura et al., 2000). Stackebrandt et al. (2001) reported $45-48 \%$ as the highest DNA-DNA relatedness values between the type strains of Nonomuraea africana, Nonomuraea dietziae and Nonomuraea recticatena, strains sharing 16S rRNA gene sequence similarities between 98.9 and $99 \cdot 8 \%$. For these reasons, it is clear that strain GW $14-1925^{\mathrm{T}}$ represents a novel species of the genus Nonomuraea, for which we propose the name Nonomuraea kuesteri. The type strain is GW $14-1925^{\mathrm{T}}$.

\section{Description of Nonomuraea kuesteri sp. nov.}

Nonomuraea kuesteri (kue'ster.i. N.L. gen. n. kuesteri of Küster, in honour of Eberhard Küster, a German microbiologist, in recognition of his numerous contributions to the taxonomy of actinomycetes).

Forms an extensive branched substrate mycelium. Only traces of aerial mycelium are visible on oatmeal agar. Spore chains are spiral; sporangia are not detected. Gram-positive and oxidase-positive; shows oxidative metabolism. Good growth occurs after 3 days incubation on oatmeal agar and nutrient agar (Oxoid) at $25-30^{\circ} \mathrm{C}$. DL-Diaminopimelic acid is the diagnostic amino acid in the peptidoglycan. The main menaquinones of the type strain are MK- $9\left(\mathrm{H}_{4}\right)$ (71\%), MK-9 $\left(\mathrm{H}_{6}\right)(15 \%), \mathrm{MK}-9\left(\mathrm{H}_{2}\right)(6 \%)$ and MK-9 $(2 \%) ; \mathrm{MK}-8\left(\mathrm{H}_{4}\right)(6 \%)$ is also present. The polar lipids include diphosphatidylglycerol, phosphatidylinositol, phosphatidylethanolamine, methyl-phosphatidylethanolamine and the diagnostic phospholipid $N$-acetylglucosamine. The fatty acids comprise mainly iso- and 


\section{Table 1. Comparison of the phenotypic properties of $N$. kuesteri sp. nov. with those of species of the genus Nonomuraea with validly published names}

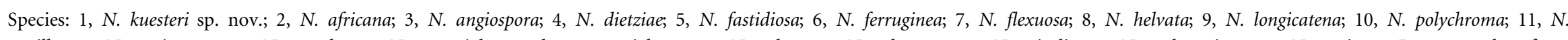

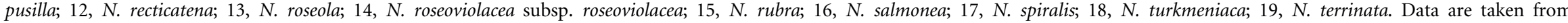

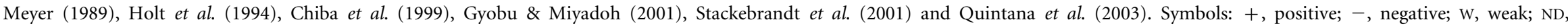
not determined.

\begin{tabular}{|c|c|c|c|c|c|c|c|c|c|c|c|c|c|c|c|c|c|c|c|}
\hline Characteristic & 1 & 2 & 3 & 4 & 5 & 6 & 7 & 8 & 9 & 10 & 11 & 12 & 13 & $14^{*}$ & 15 & 16 & 17 & 18 & 19 \\
\hline \multicolumn{20}{|l|}{ Spore-chain morphology } \\
\hline Spore chains $\dagger$ & Sp & Str & $\mathrm{Sp}$ & Str, Sp & $S, S p$ & $\mathrm{H}, \mathrm{S}$ & $\mathrm{H}, \mathrm{S}$ & $\mathrm{H}$, Psp & Str & $\mathrm{ND}$ & Psp & Str & Sp, Str & Psp & $\mathrm{H}, \mathrm{S}, \mathrm{Sp}$ & $\mathrm{H}, \mathrm{S}$ & $\mathrm{Sp}$ & $\mathrm{Sp}$ & $\begin{array}{l}\text { Irregular, } \\
\text { Psp }\end{array}$ \\
\hline Spore ornamentation & ND & Smooth & Ridged & $\begin{array}{l}\text { Cross-ridged, } \\
\text { smooth } \\
\text { and rough }\end{array}$ & Irregular & Folded & Warty & Smooth & Smooth & ND & Smooth & Smooth & Folded & Smooth & Smooth & Warty & Folded & Smooth & Rugose \\
\hline Number of spores & ND & $4-10$ & $4-15$ & $\geqslant 30$ & $4-10$ & $4-10$ & $4-10$ & $4-10$ & $10-30$ & ND & $>10$ & $4-20$ & $6-20$ & $4-20$ & $4-20$ & $4-30$ & $4-20$ & $10-20$ & $8-15$ \\
\hline \multicolumn{20}{|c|}{ Growth on ISP 3 medium } \\
\hline Aerial mycelium & Trace & $\begin{array}{l}\text { Greyish } \\
\text { blue }\end{array}$ & White & Beige & $\begin{array}{c}\text { White/ } \\
\text { pink }\end{array}$ & $\begin{array}{l}\text { White/ } \\
\text { pink }\end{array}$ & $\begin{array}{l}\text { White/ } \\
\text { yellow }\end{array}$ & White & White & Trace & $\begin{array}{l}\text { White/ } \\
\text { cream }\end{array}$ & $\begin{array}{l}\text { White/ } \\
\text { cream }\end{array}$ & Pink & $\begin{array}{l}\text { Pink/ } \\
\text { violet }\end{array}$ & Trace & Pink & $\begin{array}{l}\text { White/ } \\
\text { yellow }\end{array}$ & Trace & White \\
\hline Substrate mycelium & Yellow & Yellow & $\begin{array}{l}\text { White/ } \\
\text { ochre }\end{array}$ & Beige & Colourless & Pink & Brown & $\begin{array}{l}\text { Yellow/ } \\
\text { brown }\end{array}$ & Ochre & $\begin{array}{c}\text { Colourless/ } \\
\text { brown }\end{array}$ & $\begin{array}{l}\text { Grey/ } \\
\text { brown }\end{array}$ & $\begin{array}{c}\text { Dark } \\
\text { yellow/ } \\
\text { brown }\end{array}$ & $\begin{array}{l}\text { Brown/ } \\
\text { red }\end{array}$ & Violet & $\begin{array}{l}\text { Orange/ } \\
\text { red }\end{array}$ & Red & $\begin{array}{l}\text { Yellow/ } \\
\text { brown }\end{array}$ & $\begin{array}{c}\text { Violet/ } \\
\text { red }\end{array}$ & $\begin{array}{l}\text { White/ } \\
\text { ochre }\end{array}$ \\
\hline Soluble pigment & None & $\begin{array}{c}\text { Yellowish } \\
\text { brown }\end{array}$ & None & Yellow & None & None & None & None & None & None & None & None & None & Violet & Red & None & None & $\begin{array}{l}\text { Pink/ } \\
\text { violet }\end{array}$ & None \\
\hline \multicolumn{20}{|l|}{ Biochemical tests } \\
\hline Aesculin hydrolysis & + & + & + & ND & + & - & + & + & + & + & + & + & + & + & - & + & + & + & + \\
\hline Nitrate reductase & ND & + & - & ND & + & + & + & + & - & - & + & + & + & + & + & + & + & + & - \\
\hline \multicolumn{20}{|l|}{ Degradation of: } \\
\hline Casein & - & + & + & ND & + & + & + & - & + & - & - & - & - & - & - & + & - & + & + \\
\hline DNA & $\mathrm{ND}$ & + & + & $\mathrm{ND}$ & + & + & + & - & - & - & + & - & - & + & - & + & - & - & + \\
\hline Elastin & ND & - & + & ND & + & + & - & ND & + & + & - & + & - & - & + & + & - & - & - \\
\hline Gelatin & - & + & + & + & + & - & + & - & - & + & + & + & + & + & + & + & + & + & - \\
\hline Hypoxanthine & - & + & + & ND & + & + & - & - & + & + & + & + & + & + & + & + & - & + & + \\
\hline Starch & - & + & - & ND & - & + & + & - & + & - & - & + & - & - & + & - & - & + & + \\
\hline Tyrosine & - & + & + & ND & - & + & + & - & - & - & + & - & + & - & + & + & + & - & - \\
\hline Xanthine & - & - & - & ND & - & - & - & - & - & - & - & - & - & - & - & - & - & - & - \\
\hline \multicolumn{20}{|l|}{ Utilization of: } \\
\hline L-Arabinose & + & + & - & $\mathrm{w}$ & - & + & - & - & + & - & - & + & - & + & + & - & - & + & $\mathrm{ND}$ \\
\hline Sucrose & + & + & - & - & - & + & - & + & ND & - & - & - & + & + & + & + & + & + & $\mathrm{ND}$ \\
\hline D-Xylose & + & + & + & - & + & + & - & + & ND & - & - & - & - & - & + & - & + & + & ND \\
\hline myo-Inositol & + & + & + & - & + & - & - & + & - & - & - & + & + & + & + & - & - & + & ND \\
\hline D-Mannose & + & + & - & - & - & + & - & - & + & - & - & - & - & + & + & - & + & + & ND \\
\hline D-Fructose & + & + & - & + & - & + & - & - & $\mathrm{ND}$ & - & - & - & - & + & + & - & - & + & $\mathrm{ND}$ \\
\hline L-Rhamnose & + & + & - & + & - & + & - & - & - & - & - & + & + & + & + & + & + & + & ND \\
\hline
\end{tabular}




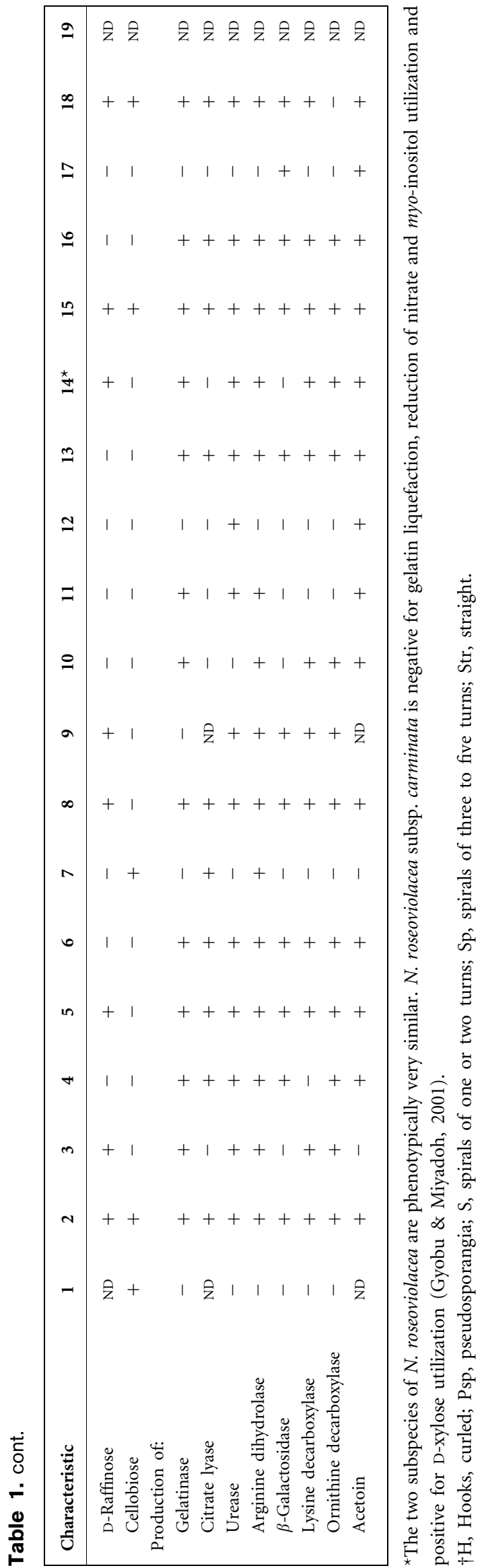

10-heptadecanoic-branched fatty acids. Smaller amounts of unsaturated and 2-hydroxy fatty acids are also detected. Results of carbon-source utilization and degradation tests (including differentiating characteristics) are shown in Table 1 . The type strain does not produce acids from the following sugars: glucose, lactose, sucrose, D-mannitol, dulcitol, salicin, adonitol, inositol, sorbitol, L-arabinose, raffinose, L-rhamnose, maltose, D-xylose, trehalose, cellobiose, methyl D-glucoside, erythritol, melibiose and arabitol. The following carbon sources are utilized (after 7 days incubation): $N$-acetyl-D-glucosamine, L-arabinose, D-cellobiose, D-fructose, D-galactose, D-glucose, D-mannose, D-melibiose, L-rhamnose, sucrose, D-trehalose, D-xylose, adonitol, D-mannitol, fumarate (weak), DL-lactate, malate, 2-oxoglutarate, L-aspartate (weak) and L-proline. The following carbon sources are not utilized: $N$-acetylD-galactosamine, $p$-arbutin, D-gluconate, D-maltose, D-ribose, salicin, inositol, maltitol, sorbitol, putrescine, acetate, propionate, cis-aconitate, trans-aconitate, adipate, 4-aminobutyrate, azelate, citrate, glutarate, DL-3-hydroxybutyrate, itaconate, mesaconate, pyruvate, suberate, Lalanine, $\beta$-alanine, L-histidine, L-leucine, L-ornithine, L-phenylalanine, L-serine, L-tryptophan, DL-3-hydroxybenzoate, DL-4-hydroxybenzotae and L-phenylacetate.

The type strain, GW $14-1925^{\mathrm{T}}\left(=\mathrm{DSM} 44753^{\mathrm{T}}=\mathrm{NRRL}\right.$ B- $24325^{\mathrm{T}}$ ), was isolated from a soil sample.

\section{References}

Chiba, S., Suzuki, M. \& Ando, K. (1999). Taxonomic re-evaluation of 'Nocardiopsis' sp. K-252 (=NRRL $15532^{\mathrm{T}}$ ): a proposal to transfer this strain to the genus Nonomuraea as Nonomuraea longicatena sp. nov. Int J Syst Bacteriol 49, 1623-1630.

Fischer, A., Kroppenstedt, R. M. \& Stackebrandt, E. (1983). Molecular-genetic and chemotaxonomic studies on Actinomadura and Nocardiopsis. J Gen Microbiol 129, 3433-3446.

Gerhardt, P., Murray, R. G. E., Wood, W. A. \& Krieg, N. R. (editors) (1994). Methods for General and Molecular Bacteriology. Washington, DC: American Society for Microbiology.

Gyobu, Y. \& Miyadoh, S. (2001). Proposal to transfer Actinomadura carminata to a new subspecies of the genus Nonomuraea as Nonomuraea roseoviolacea subsp. carminata comb. nov. Int J Syst Evol Microbiol 51, 881-889.

Holt, J. G., Krieg, N. R., Sneath, P. H. A., Staley, J. T. \& Williams, S. T. (1994). Bergey's Manual of Determinative Bacteriology, 9th edn. Baltimore: Williams \& Wilkins.

Kämpfer, P. (1990). Evaluation of the Titertek-Enterobac-Automated system (TTE-AS) for identification of Enterobacteriaceae. Zentbl Bakteriol 273, 164-172.

Kämpfer, P. \& Kroppenstedt, R. M. (1996). Numerical analysis of fatty acid patterns of coryneform bacteria and related taxa. Can J Microbiol 42, 989-1005.

Kämpfer, P., Steiof, M. \& Dott, W. (1991). Microbiological characterisation of a fuel-oil contaminated site including numerical identification of heterotrophic water and soil bacteria. Microb Ecol 21, 227-251.

Kämpfer, P., Dreyer, U., Neef, A., Dott, W. \& Busse, H.-J. (2003). Chryseobacterium defluvii sp. nov., isolated from wastewater. Int J Syst Evol Microbiol 53, 93-97. 
Kroppenstedt, R. M. (1985). Fatty acid menaquinone analysis of actinomycetes and related organisms. In Chemical Methods in Bacterial Systematics, pp. 173-199. Edited by M. Goodfellow \& D. E. Minnikin. London: Academic Press.

Kroppenstedt, R. M. \& Goodfellow, M. (1991). The family Thermomonosporaceae. In The Prokaryotes, 2nd edn, pp. 1085-1114. Edited by A. Balows, H. G. Trüper, M. Dworkin, W. Harder \& K. H. Schleifer. New York: Springer.

Kumar, S., Tamura, K., Jakobsen, I. B. \& Nei, M. (2001). MEGA2: Molecular Evolutionary Genetics Analysis software. Bioinformatics 17, 1244-1245.

Küster, E. (1959). Outline of a comparative study of criteria used in characterisation of the actinomycetes. Int Bull Bacteriol Nomencl Taxon 9, 97-104.

Lechevalier, M. P., de Bièvre, C. \& Lechevalier, H. A. (1977). Chemotaxonomy of aerobic actinomycetes: phospholipid composition. Biochem Ecol Syst 5, 249-260.

Meyer, J. (1989). Genus Actinomadura Lechevalier and Lechevalier 1970a, 400 ${ }^{\mathrm{AL}}$. In Bergey's Manual of Systematic Bacteriology, vol. 4, pp. 2511-2526. Edited by S. T. Williams, M. E. Sharpe \& J. G. Holt. Baltimore: Williams \& Wilkins.

Minnikin, D. E., O'Donnell, A. G., Goodfellow, M., Alderson, G., Athalye, M. \& Parlett, J. H. (1984). An integrated procedure for extraction of bacterial isoprenoid quinones and polar lipids. J Microbiol Methods 2, 233-241.

Poschner, J., Kroppenstedt, R. M., Fischer, A. \& Stackebrandt, E. (1985). DNA:DNA reassociation and chemotaxonomic studies on
Actinomadura, Microbispora, Microtetraspora, Micropolyspora and Nocardiopsis. Syst Appl Microbiol 6, 264-270.

Quintana, E., Maldonado, L. \& Goodfellow, M. (2003). Nonomuraea terrinata sp. nov., a novel soil actinomycete. Antonie van Leeuwenhoek 84, 1-6.

Stackebrandt, E., Wink, J., Steiner, U. \& Kroppenstedt, R. M. (2001). Nonomuraea dietzii sp. nov. Int J Syst Evol Microbiol 51, 1437-1441.

Strunk, O., Gross, O., Reichel, B. \& 10 other authors (2000). ARB: a software environment for sequence data. Munich: Department of Microbiology, Technische Universität München. http://www. arb-home.de/

Tamura, T., Suzuki, S. \& Hatano, K. (2000). Acrocarpospora gen. nov., a new genus of the order Actinomycetales. Int J Syst Evol Microbiol 50, 1163-1171.

Thompson, J. D., Gibson, T. J., Plewniak, F., Jeanmougin, F. \& Higgins, D. G. (1997). The CLUSTAL_X windows interface: flexible strategies for multiple sequence alignment aided by quality analysis tools. Nucleic Acids Res 25, 4876-4882.

Williams, S. T., Goodfellow, M., Alderson, G., Wellington, E. M. H., Sneath, P. H. A. \& Sackin, M. J. (1983). Numerical classification of Streptomyces and related genera. J Gen Microbiol 129, 1743-1813.

Zhang, Z. S., Wang, Y. \& Ruan, J. S. (1998). Reclassification of Thermomonospora and Microtetraspora. Int J Syst Bacteriol 48, 411-422.

Ziemke, F., Höfle, M. G., Lalucat, J. \& Rosselló-Mora, R. (1998). Reclassification of Shewanella putrefaciens Owen's genomic group II as Shewanella baltica sp. nov. Int J Syst Bacteriol 48, 179-186. 